\title{
Role of robotic assistance in the learning of laparoscopy: Comparison of novice students' results in two FLS trials
}

\author{
Mélanie Frigenza $^{1}$, Jérôme Delotte ${ }^{1 *}$, Eric Fontas ${ }^{2}$, Daniel Benchimol ${ }^{3}$, André Bongain ${ }^{1}$, Jean Breaud $^{4}$ \\ ${ }^{1}$ Department of Obstetrics, Gynecology, Reproductive Medicine and Fetal Medicine, University Hospital of Nice, Archet 2 Hospital, \\ Nice-Sophia Antipolis University, Nice, France \\ ${ }^{2}$ Delegation Unit for Clinical Research, Cimiez Hospital, Nice, France \\ ${ }^{3}$ General Surgery and Digestive Oncology, Archet 2 Hospital, Nice-Sophia Antipolis University, Nice, France \\ ${ }^{4}$ Department of Pediatric Surgery, Health Cooperation Group and University Hospital of Lenval, Nice-Sophia Antipolis University, \\ Nice, France \\ Email: "delotte.j@chu-nice.fr
}

Received 1 December 2012; revised 5 January 2013; accepted 16 January 2013

\begin{abstract}
Background: To investigate the learning curve of "robotic surgery" versus "conventional laparoscopy" in two trials of the FLS program in a population of medical students with no experience in laparoscopy. Methods: A prospective trial. Students, all novices in training and surgical practice, were randomized into 2 groups: the "traditional laparoscopy" group and the "robotic assistance" group. Students were evaluated during two tests from the FLS: peg transfer and intracorporeal knot tying. Results: The median laparoscopic knot completion time was significantly lower in the "robotic surgery" group $(p=0.038)$. Conclusions: Robotic assistance training in neophytes is effective in improving the completion time of laparoscopic knots.
\end{abstract}

Keywords: Da Vinci; Learning Curve; Medical Student; Laparoscopy

\section{INTRODUCTION}

Robotic surgery, an expanding surgical technique [1] that has become the "gold standard" in laparoscopic prostatectomy [2], is also developing in various surgical fields [1]. If robotic technology offers considerable benefits over traditional laparoscopy, it also imposes considerable human factor constraints on the operator, complete lack of haptic feeback by example. Nevertheless, regarding the use of this new surgical practice, Stefanidis et al. reported a benefit in the gesture all the more important than the practitioner was a novice or inexperienced in laparoscopic practice [3].

It is therefore necessary, as part of educational programs, to determine whether this benefit also exists when

\footnotetext{
${ }^{*}$ Corresponding author.
}

the practitioner has no laparoscopic experience.

The Fundamentals of Laparoscopic Surgery (FLS) certification program, established to assess the surgeons in the basic skills of laparoscopic surgery, has been proven since the late 1990s and facilitated residents' surgery training [4].

The objective of this study was to investigate the learning curve of laparoscopic surgery in conventional laparoscopy and robotics using two FLS tests in a population of medical students.

\section{MATERIALS AND METHODS}

This was a prospective single-center study, conducted at Nice University Hospital between March and April 2011. 20 students in their fifth year of medical school were recruited by random draw. The 20 students, all novices in training and surgical practice, were randomized into 2 groups of 10 each: the "traditional laparoscopy" group and the "robotic assistance" group (Figure 1).

\subsection{Sessions}

The study included four sessions for all students.

The first session, common to both groups, consisted in viewing movies on the basics of traditional and robotic laparoscopy as well as on the technical principles of two FLS tasks: peg transfer and intracorporeal knot tying.

Then, each group attended three new sessions a week apart.

During the 2nd and 4th meetings, students had to perform the two tests from the FLS: peg transfer and intracorporeal knot tying. The thread for the knot was Polysorb $^{\circledR}$ 2-0 $\left(\right.$ Syneture $\left.^{\circledR}\right)$.

The third and intermediate session tests consisted of three non-timed tests that were different from the as sessment tests of the study (cutting of a geometric form out of a compress, stacking of three cubes, handling of 


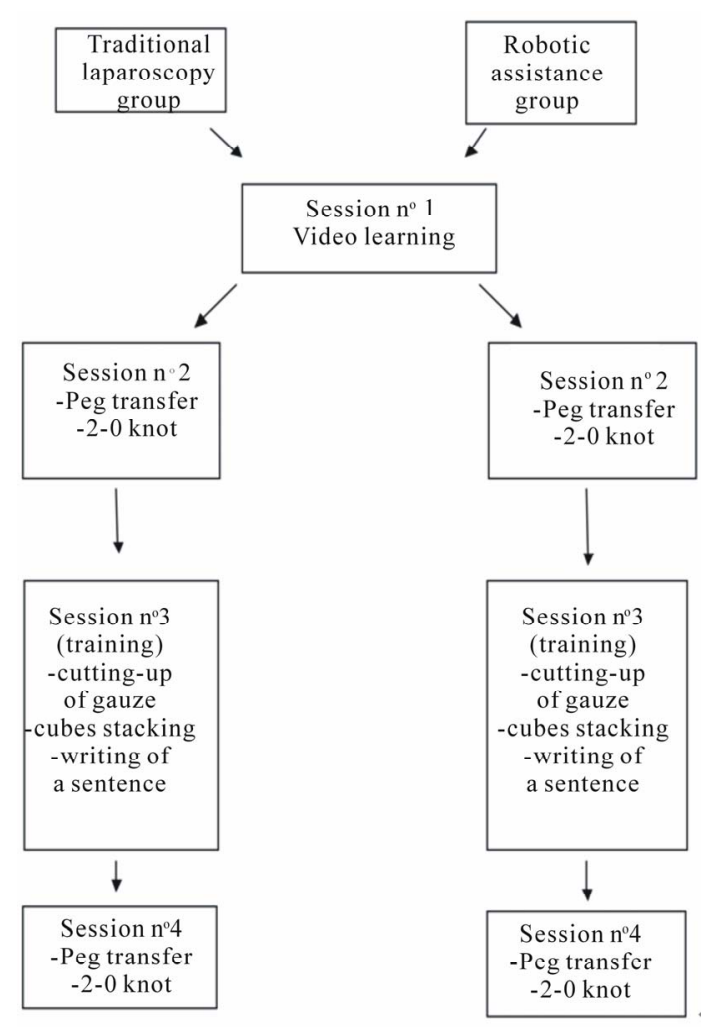

Figure 1. Algorithm for implementation of our study.

letters). The sessions of the "traditional laparoscopy" group took place in the surgical simulation center of Nice Sophia-Antipolis University, using a pelvic trainer. The sessions of the "robotic assistance" group took place in the operating room of Nice University Hospital, with the Da Vinci-S (Intuitive Surgery ${ }^{\circledR}$ ) robotic system (Figure 2).

\subsection{Data Collection}

Each student, in his own group, was allowed 5 minutes of laparoscopy or robotics equipment handling at the beginning of each test. The tests were performed in the same order for both groups, namely, first peg transfer, and second intracorporeal knot tying. Regarding the peg transfer test, two criteria were evaluated: time in seconds and the possible fall of a peg. Regarding the intracorporeal knot tying test, the considered criteria were: time in seconds, precision (assessed by the going through predefined exit points), tightening the knot, achieving three loops, and smoothness (assessed by the possible breaking of the suture material (silicone tubing)).

Students who took more than 600 seconds to perform their tests were considered as having failed and isolated in our statistical study.

\subsection{Statistics}

Statistical analysis was performed using Wilcoxon tests

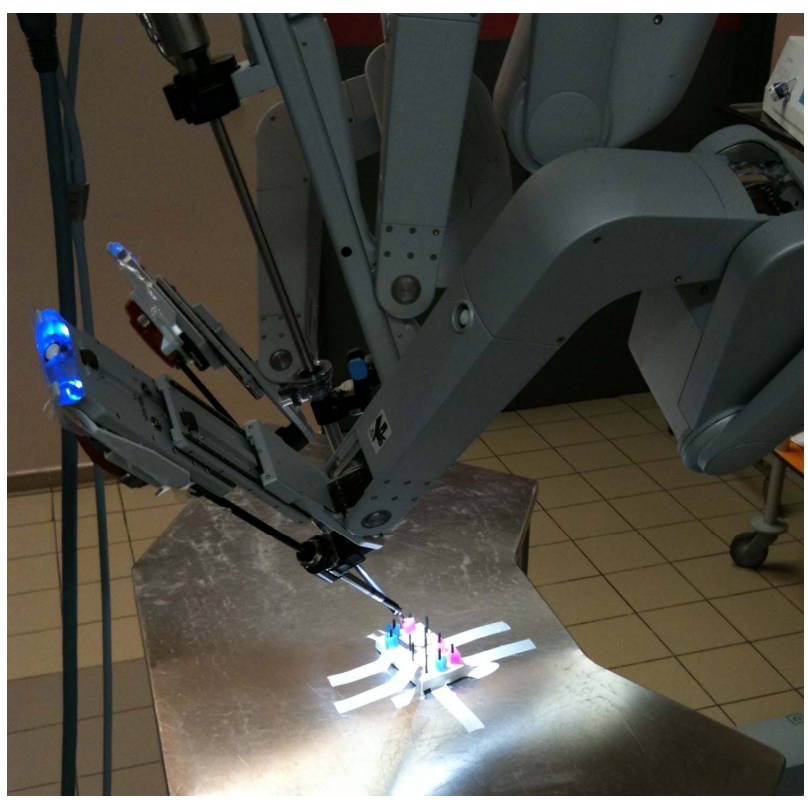

Figure 2. Cf PJ.

and McNemar for paired data, the Mann-Whitney test for comparing independent samples, and Fisher's exact test.

\section{RESULTS}

\subsection{Peg Transfer Test}

After training, the peg transfer median time was significantly reduced in both groups (Table 1).

For the same test, the comparison after training between the two groups showed an improvement of 34.9\% (i.e. 66.5 seconds) in the "traditional laparoscopy" group and $43.6 \%$ (i.e. 99 seconds) in the "robotic assistance" group (no significant difference).

3 students in the "traditional laparoscopy" group and two students in the "robotic assistance" group had the peg fall down at the 2nd session and, after training, the number of falls for each group amounted respectively to 3 and 1. There was no significant difference between both groups in the frequency of falling pegs $(p=1)$ nor within groups $(p=0.582)$.

\subsection{Intracorporeal Suturing Test}

After training, the knot tying completion median time was significantly reduced in the robotic surgery group, and not significantly in the of traditional laparoscopy group (Table 2).

For this test, the comparison after training between the 2 groups showed an improvement of 44.1\% (i.e. 142 seconds) in the conventional laparoscopy group and $31.3 \%$ (i.e. 114 seconds) in the robotic surgery group (non-significant difference, $\mathrm{p}=0.627$ ).

The study of the FLS qualitative criteria in each group 
Table 1. Peg transfer test completion time before and after training within each group.

\begin{tabular}{cccc}
\hline & $\begin{array}{c}\text { Median 2nd session } \\
\text { in seconds (min-max) }\end{array}$ & $\begin{array}{c}\text { Median 4th session } \\
\text { in seconds (min-max) }\end{array}$ & p \\
\hline $\begin{array}{c}\text { Traditional } \\
\text { laparoscopy } \\
\text { group (n=10) }\end{array}$ & $182.5(137-279)$ & $129(73-142)$ & 0.007 \\
$\begin{array}{c}\text { Robotic surgery } \\
\text { group (n= 10) }\end{array}$ & $222.5(155-455)$ & $145(117-209)$ & 0.005 \\
\hline
\end{tabular}

$\mathrm{n}=$ number of students.

Table 2. Intracorporeal knot tying completion time before and after training within each group.

\begin{tabular}{cccc}
\hline & $\begin{array}{c}\text { Median 2nd session in Median 4th session in } \\
\text { seconds (min-max) }\end{array}$ & peconds (min-max) & p \\
\hline $\begin{array}{c}\text { Traditional } \\
\text { laparoscopy } \\
\text { group (n=9) }\end{array}$ & $355(105-527)$ & $168.5(77-430)$ & 0.066 \\
$\begin{array}{c}\text { Robotic surgery } \\
\text { group }(\mathrm{n}=9)\end{array}$ & $304(178-364)$ & $198.5(133-302)$ & 0.038 \\
\hline
\end{tabular}

$\mathrm{n}=$ number of students.

before and after training is reported in Table 3.

\section{DISCUSSION}

Laparoscopy has become the gold standard in many surgical disciplines and pathologies. However, certain technical limitations (two-dimensional visualization, use of instruments with low degree of flexibility...) make its learning difficult, which is a constant concern for surgery teachers. The recent emergence of robotic assistance in laparoscopic surgery has allowed a "technological leap forward" so as to include the use of instruments more mobile than during conventional laparoscopy, the disappearance of tremor and three-dimensional vision. If the real benefits of this technology are being evaluated following indications and/or surgical disciplines, they also need to be assessed in educational programs.

In our study, intra-group comparison showed that the training session allowed students from both groups to improve significantly their speed in doing the peg transfer test.

It should be noted that to permit an assessment of all novice students, unlike the test round of the FLS, the fall of a peg was not considered as disqualifying but simply described. Anyway, training did not prove to be beneficial to any of both groups nor between groups, as far as the eventual fall of a peg in the transfer test was concerned. Completion time over 300 seconds was not considered eliminatory. Note that a significant speed increase for this task was not associated with a concomi-
Table 3. Descriptive study of the criteria of the FLS in both groups before and after training for the intracorporeal knot tying test.

\begin{tabular}{ccccc}
\hline & \multicolumn{2}{c}{$\begin{array}{c}\text { Traditional } \\
\text { laparoscopy group }\end{array}$} & \multicolumn{2}{c}{ Robotic surgery } \\
\cline { 2 - 5 } & Before & After & Before & After \\
\hline $\mathrm{n}$ & $\mathrm{n}=9$ & $\mathrm{n}=10$ & $\mathrm{n}=9$ & $\mathrm{n}=10$ \\
Knot outside the marks & 2 & 0 & 0 & 3 \\
Loose/Unsecure knot & 0 & 0 & 2 & 1 \\
Less than 3 loops achieved & 0 & 0 & 0 & 0 \\
Support ripped or torn & 0 & 0 & 0 & 0 \\
\hline $\mathrm{n}=$ number of students. & & & &
\end{tabular}

tant improvement in the number of falling pegs. We noticed that students were particularly attentive to their test timing, which allows us to suggest that these young students may have difficulties in improving their accuracy concurrently.

As regards intracorporeal knot tying, the comparisons within and between groups revealed only one significant element: a reduced completion time after training in the "robotic assistance" group. As regards the assessment of FLS qualitative criteria for the achievement of a knot, we see that the number of errors is very low, either before or after training. These results are partly explained by the fact that the evaluation criteria of the FLS were created to evaluate young surgeons still in training. Another explanation is the extra manoeuvrability afforded by robotic instrument wrists makes some of the FLS tasks probably too easy. Therefore, specific FLS tasks for robotic surgery should be determined.

The intuitive nature of the Da Vinci robot, by improving the mobility of instruments, tremor filtering and three-dimensional vision of the operative field [5], seems to reduce some technical difficulties but still requires a learning time. It can be seen as a tool for inexperienced students to learn laparoscopic gestures but still requires specific training [6]. Our study suggests, however, that this specific learning, implemented in the form of robotic assistance, seems to be rapidly successful.

It should be noted in our study that no statistical difference was demonstrated on the final results of both tests of the FLS, whether students belonged to "conventional laparoscopy" group or "robotic assistance” group. Similar results were found in the achievement of intracorporeal knots [7].

Training in simulation centers remains the basis of the teaching of laparoscopy. The development of robotic assistance will require the evaluation of specific modules of simulation or of robot learning, although the latter method seems difficult to organize because of logistical and financial constraints. The criteria of the FLS have 
appeared particularly suitable for interpreting the learning curve of novice students [8,9] even if we chose to modify certain parameters (fall of peg, more-than-300second disqualifying test) to strictly adapt to the novice status of our students.

\section{CONCLUSION}

The growing development of robotic surgery raises the question of the learning curve of this technique compared to conventional laparoscopy, especially for novice students. Using the criteria of the FLS has allowed us to demonstrate the interest for novice students of a robotic assistance-learning module for the achievement of intracorporeal knots. Because of its cost and practical constraints, the terms of roboticassisted surgery learning need to be defined.

\section{REFERENCES}

[1] Holloway, R.W., Patel, S.D. and Ahmad, S. (2009) Robotic surgery in gynecology. Scandinavian Journal of Surgery, 98, 96-109.

[2] Skolarus, T., Zhang, Y., Brent, K., et al. (2010) Robotic surgery in urologic oncology gathering the evidence. $E x$ pert Review of Pharmacoeconomics \& Outcomes Research, 10, 421-432.

[3] Stefanidis, D., Wang, F., James, R., et al. (2010) Robotic assistance improves intracorporeal suturing performance and safety in the operating room while decreasing operator workload. Surgical Endoscopy, 24, 377-382. doi:10.1007/s00464-009-0578-0

[4] Soper, N.J. and Fried, G.M. (2008) The fundamentals of laparoscopic surgery: Its time has come. Bulletin of the American College of Surgeons, 93, 30-32.

[5] Delotte, J., Mialon, O., Bouaziz, S., et al. (2011) Is there a real place for robotics in proximal tubal surgery? $G y$ necol Obstet Fertil, 39, 509-510. doi:10.1016/j.gyobfe.2011.07.001

[6] Botden, S., De Hingh, I. and Jakimowicz, J. (2009) Suturing training in augmented reality: Gaining proficiency in suturing skills faster. Surgical Endoscopy, 23, 21312137. doi:10.1007/s00464-008-0240-2

[7] De Ugarte, D., Etzioni, D., Gracia, C., et al. (2003) Robotic surgery and resident training. Surgical Endoscopy, 17, 960-963. doi:10.1007/s00464-002-8745-6

[8] Sroka, G., Feldman, L.S., Vassiliou, M.C., et al. (2010) Fundamentals of laparoscopic surgery simulator training to proficiency improves laparoscopic performance in the operating room-a randomized controlled trial. The American Journal of Surgery, 199, 115-120. doi:10.1016/j.amjsurg.2009.07.035

[9] Edelman, D.A., Mattos, M.A. and Bouwman, D.L. (2010) FLS skill retention (learning) in first year surgery residents. Journal of Surgical Research, 163, 24-28. doi:10.1016/j.jss.2010.03.057 